\title{
Electrochemical half-reaction-assisted sub-bandgap photon sensing in a graphene hybrid phsotodetector
}

\author{
Ze Xiong ${ }^{1,6}$, Jiawei Chen ${ }^{1,6}$, Jizhuang Wang ${ }^{1}, \mathrm{Yu} \mathrm{Cai}^{1}$, Xiang $\mathrm{Liu}^{2}$, Zhicheng $\mathrm{Su}^{2}$, Shijie $\mathrm{Xu}^{2}$, Arshad $\mathrm{Khan}^{3}$, \\ Wendi $\mathrm{Li}^{3}$, Juncao Bian ${ }^{4}$, Gaomin $\mathrm{Li}^{5}$, Mingyuan Huang ${ }^{5}$ and Jinyao Tang ${ }^{1}$
}

The photogating effect has been previously utilized to realize ultra-high photoresponsivity in a semiconductor-graphene hybrid photodetector. However, the spectral response of the graphene hybrid photodetector was limited by the bandgap of the incorporated semiconductor, which partially compromised the broadband absorption of graphene. Here, we show that this limitation can be overcome in principle by harnessing the electron-accepting ability of the electrochemical half-reaction. In our new graphene phototransistor, the electrochemical half-reaction serves as an effective reversible electron reservoir to accept the photoexcited hot electron from graphene, which promotes the sub-bandgap photosensitivity in a silver chloride ( $\mathrm{AgCl})$-graphene photodetector. The photoconductive gain of $~ 3 \times 10^{9}$ electrons per photon in the $\mathrm{AgCl}$-graphene hybrid is favored by the long lifetime of photoexcited carriers in the chemically reversible redox couple of $\mathrm{AgCl} / \mathrm{Ag}^{0}$, enabling a significant visible light $(400-600 \mathrm{~nm})$ responsivity that is far beyond the band-edge absorption of $\mathrm{AgCl}$. This work not only presents a new strategy to achieve an electrically tunable sub-bandgap photoresponse in semiconductor-graphene heterostructures but also provides opportunities for utilizing the electrochemical half reaction in other two-dimensional systems and optoelectronic devices. NPG Asia Materials (2017) 9, e436; doi:10.1038/am.2017.179; published online 6 October 2017

\section{INTRODUCTION}

Ultrathin two-dimensional (2D) nanomaterials are a new class of materials that have exotic properties for application in next generation electronics and optoelectronic devices. These properties include gatetunable electronic properties, high transparency, and excellent mechanical flexibility and compatibility with current silicon-based microfabrication processes. Owing to its intriguing electronic and optical properties, graphene has been studied extensively over the past decade as a promising material for broadband photodetectors. ${ }^{1-3}$ However, due to fast carrier recombination, most graphene-based photodetectors suffer from low responsivity $\left(<10 \mathrm{~mA} \mathrm{~W}^{-1}\right)$.

By contrast, graphene hybrid phototransistors ${ }^{4-8}$ provide high photoresponsivity by incorporating a layer of light absorbing material, such as a layer of semiconducting quantum dots (QDs), ${ }^{9-13}$ which is in contact with the graphene. Upon illumination, the photoexcited carriers in the QDs can be injected into graphene while the charged QDs layer can modulate the conductance of graphene by capacitive coupling. Owing to the long lifetime of injected carriers $\left(\tau_{\text {lifetime }}\right)$ in QDs, a high photoconductive gain $G\left(G=\tau_{\text {lifetime }} / \tau_{\text {transit }}\right.$, where $\tau_{\text {transit }}$ is the transit time) of $\sim 10^{8}$ can be achieved. ${ }^{9}$ Since the photon is absorbed by the incorporated light absorbing material, the spectral response of the graphene hybrid phototransistor seems to be fundamentally limited by the bandgap of the integrated materials as shown in previous studies. ${ }^{5-12}$ However, the internal photoemission from graphene in the graphene-semiconductor hybrid structure provides an alternative method to realizing broadband photo sensing. ${ }^{14-16}$ In this hybrid structure, the photocurrent is generated as the photoexcited hot electron in graphene is injected into the conduction band of the adjacent semiconductor. However, due to the low quantum efficiency and poor light absorption of graphene, only moderate photoresponsivity $\left(\sim 0.1 \mathrm{~mA} \mathrm{~W}^{-1}\right)$ was demonstrated, ${ }^{14,15}$ and signal amplification will be required at a later stage for practical application.

In this paper, we report a new graphene-semiconductor hybrid phototransistor in which the internal photoemission signal is locally amplified within the graphene transistor itself. Importantly, we show that the reversible electrochemical redox couple $\left(\mathrm{AgCl} / \mathrm{Ag}^{0}\right)$ can serve as an efficient electron reservoir to obtain an ultra-high photoconductive gain, which is the key to realizing high photoresponsivity for sub-bandgap photons in graphene hybrid phototransistors. Furthermore, we demonstrated the spectral selectivity in our graphene photodetector by simply tuning the dielectric layer thickness of the substrate, which is favorable for many color-sensitive applications. In our current design, although the spectral response is not as wide as for a PbS-graphene hybrid, in principle, it offers an alternative method to achieve broadband photosensitivity without using strongly light-

${ }^{1}$ Department of Chemistry, The University of Hong Kong, Hong Kong, China; ${ }^{2}$ Department of Physics, The University of Hong Kong, Hong Kong, China; ${ }^{3}$ Department of Mechanical Engineering, The University of Hong Kong, Hong Kong, China; ${ }^{4}$ Department of Physics and Materials Science, City University of Hong Kong, Hong Kong, China and ${ }^{5}$ Department of Physics, South University of Science and Technology of China, Shenzhen, China

${ }^{6}$ These authors contributed equally to this work.

Correspondence: Dr J Tang, Department of Chemistry, University of Hong Kong, Pokfulam, Hong Kong 999077, China.

E-mail: jinyao@hku.hk

Received 8 May 2017; revised 21 July 2017; accepted 18 August 2017 
absorbing narrow-band semiconductor materials, which shows promise for highly transparent photodetectors and other novel optoelectronic devices.

\section{METHODS}

\section{Photodetector device fabrication}

The process flow of the AgX-graphene hybrid photodetector fabrication is illustrated in Supplementary Figure 1. Monolayer chemical vapor deposition (CVD) graphene on a copper foil (Graphene Laboratories, Inc., Calverton, NY, USA) was spin-coated with poly(methyl methacrylate) (PMMA) and air dried. Then, the copper was removed by etching in $0.1 \mathrm{M}\left(\mathrm{NH}_{4}\right)_{2} \mathrm{~S}_{2} \mathrm{O}_{8}$ aqueous solution overnight. The released graphene layer that was attached on the PMMA film was scooped out and rinsed consecutively in several clean DI water baths. Finally, the film was transferred onto various supporting substrates (silicon wafer, a quartz substrate or polyethylene terephthalate (PET) film) and air dried. After dissolving PMMA with a mixture solution of dichloroethane and acetone $(1: 5, \mathrm{v} / \mathrm{v})$, the graphene was patterned into micro-ribbons with electron beam lithography followed by oxygen plasma etching. Then, $0.5-\mathrm{nm}$ silver was deposited on the lithographically defined central area of the graphene ribbon via thermal evaporation in vacuum at $\sim 1 \times 10^{-6}$ Torr.

Vapor-solid reactions were utilized to convert the Ag nanoparticles into the $\mathrm{AgCl}$ nanoparticles. A 5-ml glass vial with the sample substrate was placed in a sealed $25-\mathrm{ml}$ glass vial together with $0.05 \mathrm{~g}$ of $\mathrm{KClO}_{3}$ solid crystals. Then, $0.15 \mathrm{ml}$ of $37 \% \mathrm{HCl}$ solution was added to the solid $\mathrm{KClO}_{3}$, and the $\mathrm{Cl}_{2}$ was generated via an in situ reaction $\left(\mathrm{KClO}_{3}+6 \mathrm{HCl}=\mathrm{KCl}+3 \mathrm{Cl}_{2}+3 \mathrm{H}_{2} \mathrm{O}\right)$ and allowed to react with $\mathrm{Ag}$ nanoparticles on the sample substrate for $10 \mathrm{~min}$ at room temperature. For the $\mathrm{AgBr}$ nanoparticle synthesis, a 5-ml glass vial with the sample substrate was placed in a sealed $25-\mathrm{ml}$ glass vial with $0.05 \mathrm{~g}$ of solid $\mathrm{KBr}$. Then, $0.15 \mathrm{~g}$ of solid $\mathrm{KMnO}_{4}$ and $98 \% \mathrm{H}_{2} \mathrm{SO}_{4}$ solution were added to the solid, and $\mathrm{Br}_{2}$ vapor was produced via reaction $\left(2 \mathrm{KMnO}_{4}+8 \mathrm{H}_{2} \mathrm{SO}_{4}+10\right.$ $\mathrm{KBr}=6 \quad \mathrm{~K}_{2} \mathrm{SO}_{4}+5 \quad \mathrm{Br}_{2}+2 \quad \mathrm{MnSO}_{4}+8 \quad \mathrm{H}_{2} \mathrm{O}$ ) and allowed to react with $\mathrm{Ag}$ nanoparticles for $10 \mathrm{~min}$ at room temperature. For the AgI nanoparticle synthesis, the sample substrate was placed in a sealed $25-\mathrm{ml}$ glass vial containing $0.05 \mathrm{~g}$ of solid $\mathrm{I}_{2}$. Then, the reaction vial was heated to $105^{\circ} \mathrm{C}$ to allow the $\mathrm{I}_{2}$ vapor to react with $\mathrm{Ag}$ nanoparticles for $10 \mathrm{~min}$. After the vapor-solid conversion, 5- $\mathrm{nm} \mathrm{Al}_{2} \mathrm{O}_{3}$ was coated using atomic layer deposition (Cambridge NanoTech, Inc., Waltham, MA, USA, Savannah 200) using trimethylaluminum (Sigma-Aldrich, Inc., St Louis, MO, USA) as the precursor at $70{ }^{\circ} \mathrm{C}$. The $\mathrm{Al}_{2} \mathrm{O}_{3}$ on the electrical contact area was removed by dipping in a 5:1 buffered HF solution for $\sim 1 \mathrm{~s}$. The electrodes were patterned on the graphene ribbon using electron beam lithography, followed by $100-\mathrm{nm}$ nickel $(\mathrm{Ni})$ sputtering.

\section{Transmission electron microscope (TEM) sample preparation}

As shown in Supplementary Figure 3, the monolayer CVD graphene on copper was spin-coated with PMMA and air dried. After copper removal, the graphene film was rinsed in DI water and scooped onto the TEM grid (SPI Supplies, Inc., West Chester, PA, USA, G200HS) with a $5-\mathrm{nm} \mathrm{Al}_{2} \mathrm{O}_{3}$ coating produced via atomic layer deposition. Then, $0.5-\mathrm{nm}$ Ag was deposited through the mesh of the grid onto graphene via thermal evaporation and individually reacted with $\mathrm{Cl}_{2}, \mathrm{Br}_{2}$ and $\mathrm{I}_{2}$. After the reaction, another $5 \mathrm{~nm}$ of $\mathrm{Al}_{2} \mathrm{O}_{3}$ was coated over the device via atomic layer deposition at $70{ }^{\circ} \mathrm{C}$ to protect the $\mathrm{AgX}$-graphene surface.

\section{KPFM measurement}

The device with graphene on top of the $\mathrm{AgCl}$ nanoparticles was prepared using a TEM grid-assisted dry transfer method similar to the TEM sample preparation. The monolayer CVD graphene supported with PMMA film was scooped by the $\mathrm{Al}_{2} \mathrm{O}_{3}$-coated TEM grid. After the $\mathrm{AgCl}$ conversion reaction, PMMA-supported graphene was transferred onto a clean Si substrate with $470 \mathrm{~nm}$ of a thermal oxide and baked on the hot plate at $120^{\circ} \mathrm{C}$ for $5 \mathrm{~min}$ to improve the adhesion between the graphene and the substrate. Then, the PMMA on graphene was removed by rinsing with a mixture solution of dichloroethane and acetone $(1: 5, \mathrm{v} / \mathrm{v})$, followed by isopropanol rinsing and blow drying with nitrogen. A $15-\mathrm{nm}$ Au electrode was patterned on the graphene surface as a work function reference via electron beam lithography and thermal evaporation. The KPFM measurement was performed using an Asylum Research MFP-3D-SA atomic force microscope under ambient conditions. Asylum Research ASYELEC-01 cantilevers with titanium and iridium coatings $(3 \mathrm{~nm} / 17 \mathrm{~nm})$ were used at a resonance frequency of $72.394 \mathrm{kHz}$ with a tip voltage of $+3.0 \mathrm{~V}$ with respect to the ground. The illumination during scanning was achieved by utilizing a Fiber-Liter MI-150R illuminator with a remote controller (Dolan-Jenner Industries, Boxborough, MA, USA).

\section{Photoresponse measurements}

The temporal photoresponse and gate tunability of the AgX-G hybrid device were measured using a white light LED light source with an adjustable power output. The spectral response of the hybrid photodetector was measured using an SVX 1450 xenon lamp light source (Müller Elektronik-Optik, Moosinning, Germany) and an Acton SP 300i monochromator equipped with 1200 lines per $\mathrm{mm}$ of grating. The photocurrents of the samples were measured using a Keithley 2400 Source Meter. The eEQE was measured with a super continuum laser (YSL Photonics, Wuhan, Hubei, China, Supercontinuum Source SC-PRO) with a laser pulse of $\sim 100 \mathrm{ps}$ and a repetition rate of $3 \mathrm{MHz}$. The photoresponsivities at 400 and $500 \mathrm{~nm}$ were measured using a monochromatic LED to provide uniform illumination. All light source intensities were calibrated using a standard silicon diode (Hamamatsu S1336-BQ) and an optical power meter (Molectron Detector, Inc. Portland, OR, USA, POWER MAX 500D).

\section{Finite-difference-time-domain simulation}

The power absorption spectrum of the AgCl-graphene heterostructure was calculated using a finite-difference-time-domain software package (Lumerical Solutions, Inc., Vancouver, BC, Canada, FDTD Solutions). Since the nanoparticles were well dispersed, a single $\mathrm{AgCl}$ sphere with a diameter of $18 \mathrm{~nm}$ on monolayer graphene was modeled, which showed no significant difference from the model with multiple $\mathrm{AgCl}$ spheres. A 5- $\mathrm{nm} \mathrm{Al}_{2} \mathrm{O}_{3}$ capping layer was also included in the model. The optical constants of $\mathrm{Al}_{2} \mathrm{O}_{3}, \mathrm{SiO}_{2}$ and $\mathrm{Si}$ were selected from the software database (Palik, E.D., Handbook of Optical Constants). The absorption simulation was performed with a cross-section analysis module, total-field scattered-field light source and perfectly matched layers boundary conditions without light polarization.

\section{RESULTS}

Photodetector design, device fabrication and temporal photoresponse

We fabricated the phototransistors using a silver halide-graphene hybrid (AgX-G, $\mathrm{X}=\mathrm{Cl}, \mathrm{Br}, \mathrm{I}$ ) as depicted in the schematic diagram (Figure 1a). Degenerately p-doped Si substrate with 470-nm thermal oxide was used as the back-gate electrode to modulate the Fermi level of the AgX-G hybrid. Well-dispersed silver nanoparticles were deposited on the CVD graphene via thermal evaporation (see Methods and Supplementary Figure 1). Then, the Ag nanoparticles were completely converted into AgX nanoparticles by allowing the $\mathrm{Ag}$ nanoparticles to react with the respective halogen gas $\left(2 A g_{(s)}+X_{2(g)} \rightarrow 2 A g X_{(s)}\right)$ as observed in X-ray photoelectron spectroscopy (Supplementary Figure 2). After the reaction, the AgX nanoparticles were well dispersed on the graphene surface (Supplementary Figures 3 and 4) with an average diameter of $18 \mathrm{~nm}$. Finally, 5-nm $\mathrm{Al}_{2} \mathrm{O}_{3}$ was coated over the device surface with atomic layer deposition to minimize the influence of ambient gas molecule adsorption and desorption upon illumination. To estimate the possible contribution of the plasmonic absorption from the trace amount of unreacted Ag metal on the graphene surface, a control device was fabricated with silver nanoparticles over the graphene surface. Because no detectable photoresponse was observed in this control sample (Supplementary Figure 5), it was safe to conclude that the Ag metal plasmonic contribution to the photoresponse in our device was insignificant. 
a

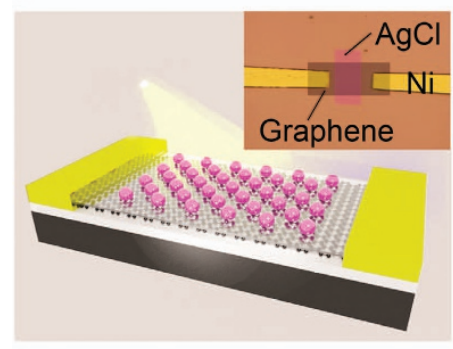

d

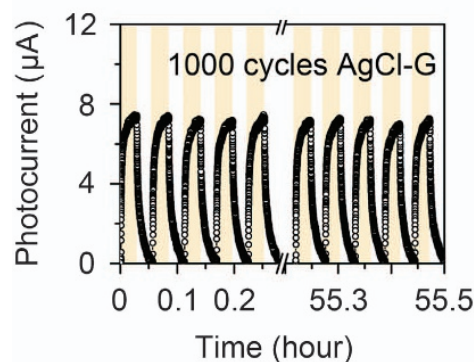

b

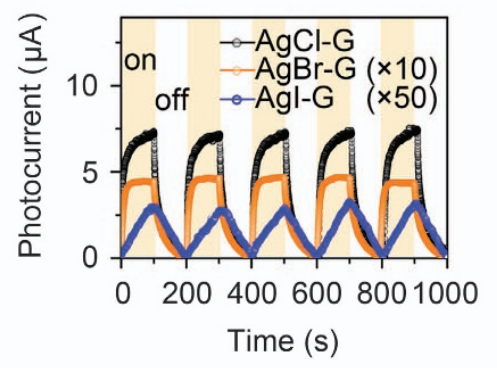

C

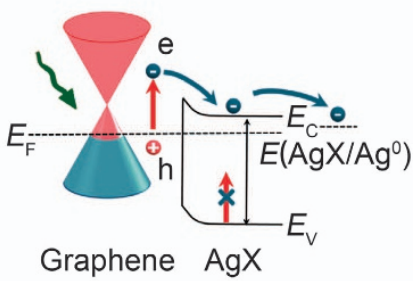

e

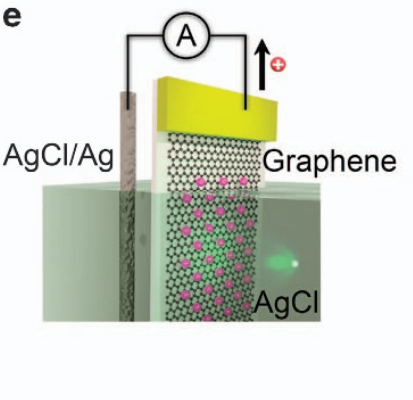

f



Figure $1 \mathrm{AgCl}$-graphene photodetector and temporal photoresponse. (a) A schematic diagram of the silver halide-graphene (AgX-G, $\mathrm{X}=\mathrm{Cl}$, Br, I) photodetector. Inset: A false colored optical image of the as-prepared AgCl-G photodetector. (b) The temporal photoresponse of the as-prepared AgX-G hybrid photodetectors. The visible light illumination power is $54.9 \mathrm{nW}$, and the source-drain voltage $\left(V_{\mathrm{SD}}\right)$ is $1 \mathrm{~V}$. The photocurrents of $\mathrm{AgBr}-\mathrm{G}$ and $\mathrm{Agl}-\mathrm{G}$ have been magnified by 10 and 50 times, respectively, for clarity. (c) The energy band diagram at the junction formed by graphene and the semiconducting AgX. For low-energy photons, the AgX cannot be excited directly due to its wide bandgap $\left(E_{\mathrm{g}}^{\mathrm{AgCl}}=3.25 \mathrm{eV}, E_{\mathrm{g}}^{\mathrm{AgBr}}=2.69 \mathrm{eV}, E_{\mathrm{g}}^{\mathrm{Agl}}=2.83 \mathrm{eV}\right) .^{28} \mathrm{Instead}$, the hot electrons generated in graphene are injected into the conduction band of the AgX nanoparticles through internal photoemission and transferred to the redox level of $\mathrm{AgX/Ag}^{0}$. (d) The long-time (1000 cycles) temporal photoresponse of the $\mathrm{AgCl}-\mathrm{G}$ photodetector after 6 months of preservation under ambient conditions. (e) The schematic diagram of the photoelectrochemical measurement setup for internal photoemission at the AgCl-graphene junction. (f) The temporal photoelectrochemical response of the AgCl-G working electrode at a 0-V bias under chopped green light illumination ( $520 \mathrm{~nm}$ wavelength).

We examined the photoresponse of hybrid phototransistors with $\mathrm{AgCl}, \mathrm{AgBr}$ or $\mathrm{AgI}$ under the same visible illumination. Since $\mathrm{AgCl}$ is a wide bandgap $\left(E_{\mathrm{g}}^{\mathrm{AgCl}}=3.25 \mathrm{eV}\right)$ semiconductor, only ultraviolet sensitivity is expected (Supplementary Figure 6), assuming the same sensing mechanism as previously reported for a graphenesemiconductor hybrid phototransistor. ${ }^{9-13}$ By contrast, a high photoresponse was observed with visible light illumination, suggesting a new sensing mechanism in our phototransistor. Figure $1 \mathrm{~b}$ shows the photocurrent (after dark current subtraction) of the as-prepared $\mathrm{AgX}-\mathrm{G}$ photodetectors at room temperature under chopped light illumination (white light illumination with a power of $\sim 54.9 \mathrm{nW}$ ) at a $1-\mathrm{V}$ source-drain voltage $\left(V_{\mathrm{SD}}\right)$ and a $0-\mathrm{V}$ back-gate voltage $\left(V_{\mathrm{G}}\right)$. If working as a traditional graphene hybrid phototransistor in which the semiconductor is responsible for light absorption, the photoresponse of the AgI-G hybrid should be the highest because AgI has the lowest bandgap and the highest photosensitivity. However, the observed photoresponse of the AgCl-G hybrid is much stronger than that of AgBr-G and AgI-G, which strongly suggests that the photoresponse did not originate from $\mathrm{AgX}$ nanoparticle absorption. To explain this phenomenon, we attributed this sub-bandgap photosensitivity in our device to the electrochemically assisted internal photoemission from graphene to the AgX nanoparticles. As illustrated in Figure 1c, upon illumination with low-energy photons, the photoexcited hot carriers with an energy higher than the Schottky barrier height $\left(\Phi_{\mathrm{B}}\right)$ can be injected into the $\mathrm{AgX}$ nanoparticles ${ }^{17}$ and then stored into the low-energy redox level of $\mathrm{AgX} / \mathrm{Ag}^{0}$ by inducing the half electrochemical reduction reaction $\mathrm{AgX}+e^{-} \rightarrow \mathrm{Ag}^{0}+X^{-}$, which will negatively charge the AgX nanoparticles and enhance the conductance of the graphene transistor via capacitive coupling. In the dark, the reduction reaction is reversed and the injected electron is released back to graphene through a tunneling process, restoring the phototransistor back to its low conductance state. Here, the sub-bandgap photoresponsivity of the graphene hybrid detector is primarily determined by the nanoparticle's ability to accept electrons. In electrochemistry, the electrochemical potential $(E)$ is classically used to represent the ability of an oxidant to accept electrons. In our case, since $\mathrm{AgCl}$ is a stronger oxidant than $\mathrm{AgBr}$ and $\mathrm{AgI}\left(E_{\mathrm{AgCl} / \mathrm{Ag}}=-4.66 \mathrm{eV}<E_{\mathrm{AgBr} / \mathrm{Ag}}=\right.$ $\left.-4.51 \mathrm{eV}<E_{\mathrm{Ag} / \mathrm{Ag}}=-4.29 \mathrm{eV}\right)^{18}$ and readily retains the injected hot carriers from the graphene, the highest gain and photoresponsivity were observed in AgCl-G, followed by AgBr-G and AgI-G. This result suggested that the classical standard electrochemical potential could also be used to benchmark the electron-accepting ability of other electrochemical reaction-assisted graphene hybrid phototransistors for low energy photon sensing. Without a favorable redox couple (for example, in the $\mathrm{ZnO}$-graphene hybrid), the internal photoemission from graphene to the semiconductor is insignificant and the sub-bandgap photoresponse is negligible. ${ }^{10,11}$ Since our phototransistor involves an electrochemical redox reaction, it is important to assess the stability of our device, which is determined by the reversibility of the redox reaction. The AgCl-G sample was stored under ambient conditions for 6 months and then subjected to a long stress test under chopped illumination (Figure 1d). After over 1000 on-off cycles, the photocurrent maintained the same level, indicating the great reversibility and reliability of our device.

To confirm the sensing mechanism in our phototransistor, we prepared a photoelectrochemical electrode based on the AgCl-G hybrid to test the internal photoemission current from graphene to the $\mathrm{AgCl}$ nanoparticles at a $0-\mathrm{V}$ bias. As shown in Figure $1 \mathrm{e}, \mathrm{AgCl}$ 
a

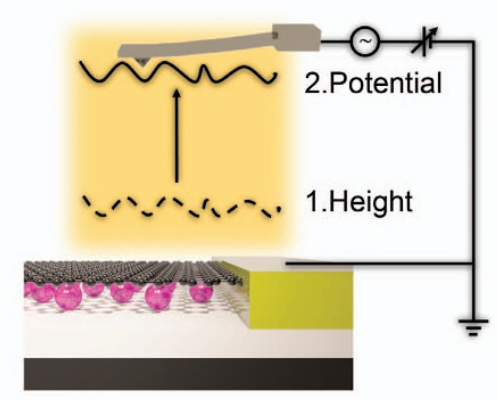

c

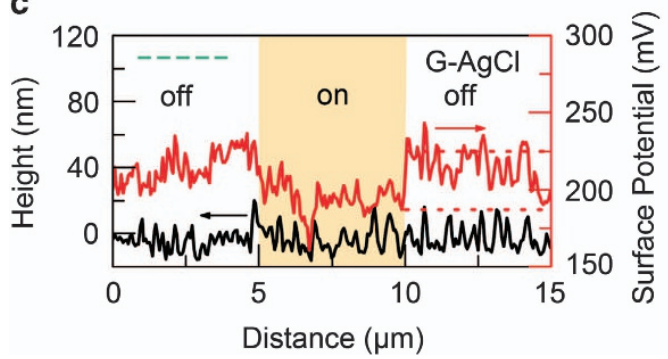

b
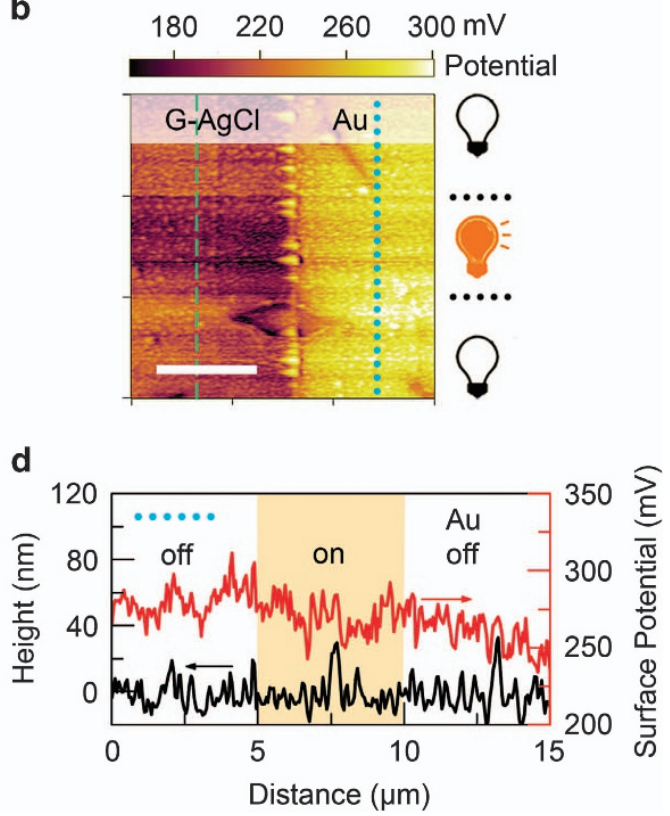

Figure 2 Structure and surface potential profiles of graphene-AgCl. (a) KPFM setup for the two-pass technique. (b) KPFM image taken on the surface of graphene-AgCl (left) and the Au electrode (right) under alternating 'dark', 'light' and 'dark' illumination states at $V_{\mathrm{G}}=0 \mathrm{~V}$. Scale bar: $5 \mu \mathrm{m}$. (c) Height and surface potential profiles along the green dashed lines on the graphene-AgCl surface. (d) Height and surface potential profiles along the blue dotted lines on the Au surface. KPFM, Kelvin probe force microscopy.

nanoparticle-loaded monolayer graphene was used as a working electrode and submerged into a $1 \mathrm{M} \mathrm{KCl}$ solution with $\mathrm{AgCl} / \mathrm{Ag}$ as the counter electrode. Upon illumination with low-energy photons $(520 \mathrm{~nm})$, the $\mathrm{AgCl}$ could not be excited while the hot electron was generated in graphene. We recorded the temporal photocurrent at the $0-\mathrm{V}$ bias in chopped green light illumination, as shown in Figure 1f. Since no detectable photocurrent was observed for $\mathrm{AgCl}$ nanoparticles over the platinum surface (Supplementary Figure 7), which has a similar work function as graphene $\left(\Phi_{\text {graphene }}=4 . \tilde{95.1}, \Phi_{\mathrm{Pt}}=5.1\right),{ }^{19}$ the photocurrent clearly did not originate from the $\mathrm{AgCl}$ absorption and should be attributed to the graphene internal photoemission similar to the recently demonstrated graphene-semiconductor photodetector. ${ }^{14,15}$ In this mechanism, since the internal photoemission is controlled by the Schottky barrier height at the graphenesemiconductor interface instead of the bandgap of the incorporated semiconductor, our photodetector is capable of exploiting subbandgap photons and extending the sensitivity to the visible spectrum. This strategy offers a new approach to engineer the spectral response of optoelectronic devices via surface engineering without using narrow bandgap materials.

\section{KPFM characterization}

To further verify the internal photoemission process at the $\mathrm{AgCl}$ graphene junction, Kelvin probe force microscopy (KPFM) was utilized to investigate the surface potential modulation of graphene under different illumination conditions (Supplementary Figure 8). Because the KPFM measurement is highly surface-sensitive, a sample with graphene on top of $\mathrm{AgCl}(\mathrm{G}-\mathrm{AgCl}$ ) was prepared (see Methods). As shown in Figures $2 a$ and $b$, the surface potential image was taken using the standard two-pass technique while the electrically grounded Au electrode was used as an internal reference to show the surface potential change upon illumination.

The surface potential in the KPFM measurement is determined by the contact potential difference between the probe and the sample surface. Given the identical probe conditions, the difference in the work function between the $\mathrm{Au}$ electrode $\left(\Phi_{\mathrm{Au}}\right)$ and the G-AgCl $\left(\Phi_{\mathrm{g}}\right)$ can be related to the surface potential difference, $\Delta V_{\mathrm{CPD}}$, as $e \Delta V_{\mathrm{CPD}}=e\left(V_{\mathrm{g}}-V_{\mathrm{Au}}\right)=\Phi_{\mathrm{Au}}-\Phi_{\mathrm{g}}{ }^{20}$ where $V_{\mathrm{Au}}$ and $V_{\mathrm{g}}$ are the average surface potential of the $\mathrm{Au}$ electrode and the $\mathrm{G}-\mathrm{AgCl}$, respectively. Hence, the work function of graphene can be quantified after taking the work function of the electrically grounded $\mathrm{Au}$ electrode $\left(\Phi_{\mathrm{Au}}=4.82 \mathrm{eV}\right){ }^{21}$ The surface potential profiles in Figures $2 \mathrm{c}$ and $\mathrm{d}$ show that the work function of $\mathrm{G}-\mathrm{AgCl}$ upshifts from a 'dark state' $\left(\Phi_{\mathrm{g}}^{\mathrm{dark}} \approx 4.88 \mathrm{eV}\right)$ to a 'light state' $\left(\Phi_{\mathrm{g}}^{\text {light }} \approx 4.90 \mathrm{eV}\right)$, whereas no detectable shift in work function was observed on the Au electrode. Additionally, there was no distinguishable potential change after visible light illumination in the control sample made of graphene without $\mathrm{AgCl}$ nanoparticles (Supplementary Figure 9c), which indicates that the photoexcited hot electrons were emitted from graphene to the $\mathrm{AgCl}$ nanoparticles upon illumination. The photoactive area in our $\mathrm{AgCl}-\mathrm{G}$ device is not constrained to the vicinity of the Au electrode, which allows a larger photoactive area for photon harvesting than that of photovoltaic graphene photodetectors. ${ }^{1,2}$

\section{Sub-bandgap photoresponse of the AgCl-graphene hybrid}

The performance of the AgCl-G phototransistor under visible illumination was further explored in detail. Figure 3a shows the dependence of responsivity on $V_{\mathrm{SD}}$ under 500-nm illumination with different powers at $V_{\mathrm{G}}=0 \mathrm{~V}$ (400 nm illumination, see Supplementary Figure 10). The raised responsivity at higher $V_{\mathrm{SD}}$ values can be ascribed to the shortened transit time and the subsequently improved gain value. Figure $3 \mathrm{~b}$ plots the responsivity as a function of the light power for 500-nm illumination at $V_{\mathrm{SD}}=1 \mathrm{~V}$ and $\mathrm{V}_{G}=0 \mathrm{~V}$. A high responsivity of $\sim 7.3 \times 10^{4} \mathrm{AW}^{-1}$ was obtained under an illumination power of $16.4 \mathrm{pW}$. Under a higher illumination intensity, the photoresponsivity gradually decreases because the filling of the $\mathrm{AgCl} / \mathrm{Ag}^{0}$ reservoir prevents the accepting of additional photocarriers and shortens the average $\tau_{\text {lifetime. }}{ }^{10}$ 
a

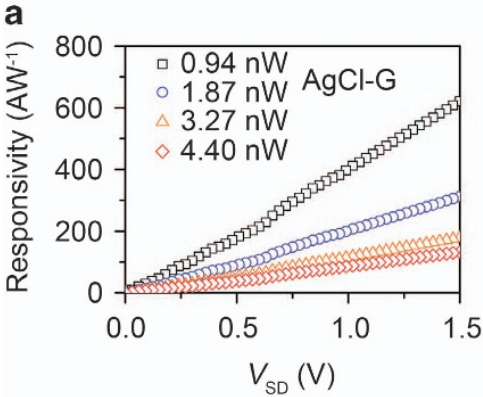

d

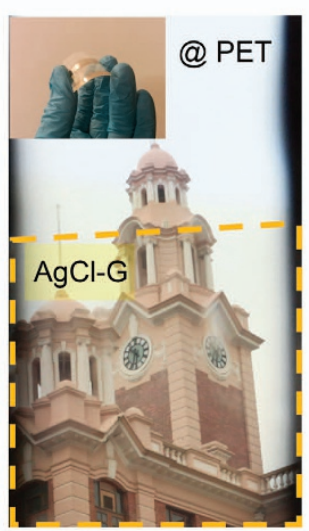

b

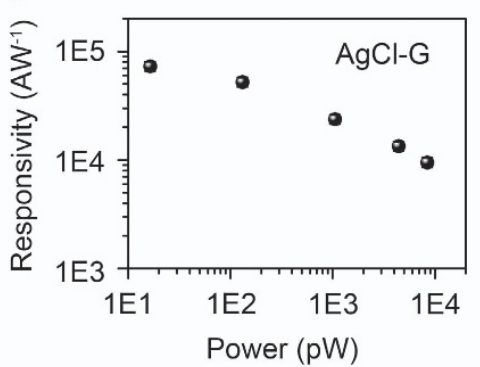

e

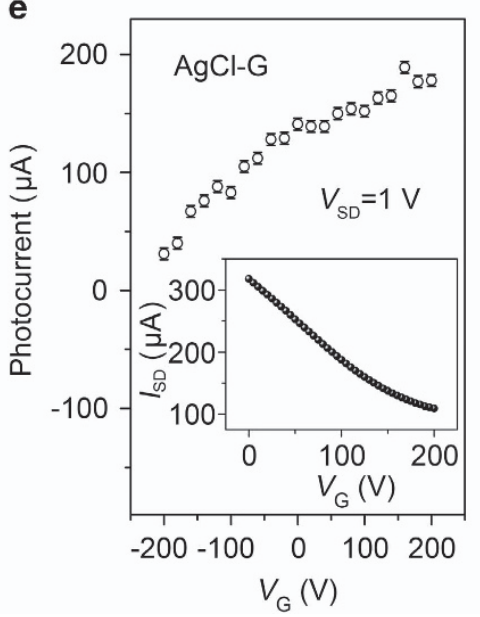

C

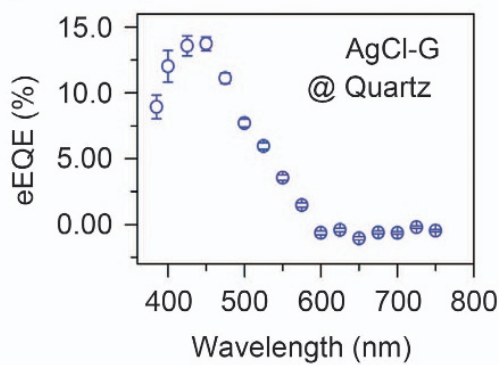

f
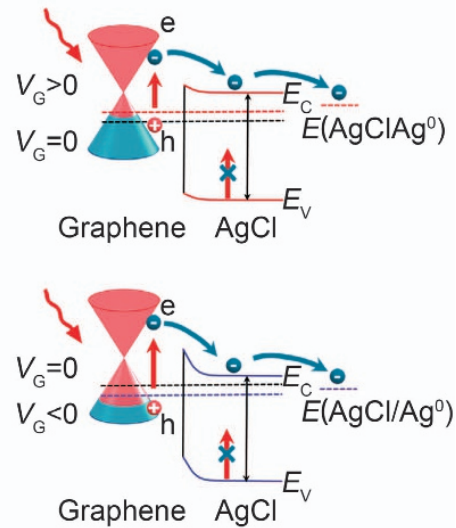

Figure 3 Sub-bandgap and gate-tunable photoresponse. (a) The photoresponsivity increases linearly with $V_{S D}$ under $500-n m$ illumination of different optical input powers at $V_{G}=0 \mathrm{~V}$. (b) Photoresponsivity as a function of the optical input power under $500-\mathrm{nm}$ illumination at $V_{\mathrm{SD}}=1$ and $V_{\mathrm{G}}=0 \mathrm{~V}$. (c) The effective external quantum efficiency (eEQE) of the AgCl-G sample on quartz at $V_{S D}=1 \mathrm{~V}$. (d) A photo of the University's main building taken through flexible AgCl-G (the area of $\mathrm{AgCl}-\mathrm{G}$ is highlighted by a brown dashed line) on PET film. Inset: A photographic image of the flexible AgCl-G photodetector on PET film. (e) The photocurrent of $\mathrm{AgCl}-\mathrm{G}$ as a function of $V_{\mathrm{G}}$ under 500-nm light illumination with a power of $\sim 5.42 \mathrm{nW}$ at $V_{\mathrm{SD}}=1 \mathrm{~V}$. Inset: Transfer characteristics of the $\mathrm{AgCl}-\mathrm{G}$ phototransistor in the dark state, showing p-type doping in graphene by $\mathrm{AgCl}$ nanoparticles. (f) Energy band diagram of the AgCl-G phototransistor at different $V_{G}$ values. The dashed lines correspond to the Fermi level of graphene at various $V_{G}$ (black for $V_{G}=0$, red for $V_{G}>0$ and blue for $V_{G}<0$ ) values.

To evaluate the intrinsic spectral response of AgCl-G, the effective external quantum efficiency (eEQE) was estimated by multiplying the external quantum efficiency by the photoconductivity gain of the device (Figure 3c)..$^{5} \mathrm{~A}$ AgCl-G sample on a quartz substrate was measured with monochromatic illumination. The eEQE had a pronounced value in the visible range from 385 to $600 \mathrm{~nm}(13.6 \%$ at $425 \mathrm{~nm}$ ) beyond the band edge of $\mathrm{AgCl}$. As predicted by the Fowler theory, ${ }^{22}$ hot carriers with lower energy have less photoemission probability, which leads to the decreasing of eEQE towards a longer wavelength. When the light wavelength goes below $425 \mathrm{~nm}$, the photoexcited electrons from $\mathrm{AgCl}$ itself will partially occupy the $\mathrm{AgCl} / \mathrm{Ag}^{0}$ redox couple, hindering the collection of photocarriers from graphene and lowering the eEQE. Owing to the low light absorbance of the graphene and the ultrathin AgCl layer, a transparent AgCl-G phototransistor with a high transmittance of $\sim 92.8 \%$ could be prepared on PET film (Figure 3d).

The carrier mobility $(\mu)$ of the graphene channel in AgCl-G without illumination was calculated to be $362.2 \mathrm{~cm}^{2} \mathrm{~V}^{-1} \mathrm{~s}^{-1}$ from the transfer characteristic measurement (Figure $3 \mathrm{e}$, inset) using $\mu=\left(\mathrm{d} I_{\mathrm{SD}} / \mathrm{d} V_{\mathrm{G}}\right) \times\left[L /\left(W C_{i} V_{\mathrm{SD}}\right)\right]$, where $L$ is the channel length $(22 \mu \mathrm{m}), W$ is the channel width $(16 \mu \mathrm{m})$ and $C_{i}$ is the areal capacitance $\left(7.34 \mathrm{nF} \mathrm{cm}^{-2}, \mathrm{SiO}_{2}\right.$ thickness of $\left.470 \mathrm{~nm}\right){ }^{23}$ The gate tunability of the photocurrent in the AgCl-G device is shown in Figure $3 \mathrm{e}$ under a uniform 500 -nm light illumination with a power of $\sim 5.42 \mathrm{nW}$ at $V_{\mathrm{SD}}=1.0 \mathrm{~V} \quad(385 \mathrm{~nm}$ light illumination see
Supplementary Figure 11). As shown in Figure $3 \mathrm{f}$, a negative $V_{\mathrm{G}}$ leads to a lower Fermi level in graphene and a raised barrier height at the AgCl-graphene junction, which mitigates the hot electron injection into $\mathrm{AgCl}$ and leads to lower overall photoresponsivity.

\section{Spectral selectivity modulation}

The spectral selectivity in graphene has previously been realized by integrating with various photonic devices via sophisticated fabrication processes. ${ }^{24-26}$ We demonstrate that, in our phototransistor, the spectral selectivity can be accomplished by simply modulating the thickness of the thermal oxide underneath the graphene layer (Figure 4a). As shown in Figure 4 b, for $\mathrm{AgCl}-\mathrm{G}$ on a silicon substrate with $470 \mathrm{~nm}$ of $\mathrm{SiO}_{2}$, two major responsivity peaks at 400 and $500 \mathrm{~nm}$ were observed. This enhancement can be attributed to the optical resonance inside the dielectric layer, which resembled the spectral response of single-layer graphene on $300-\mathrm{nm} \mathrm{SiO}_{2}{ }^{3}$. The power absorption spectrum predicted by the finite-difference-time-domain simulation matches well with the experimental data. As a comparison, only a single photoresponse peak located at approximately $400 \mathrm{~nm}$ could be identified for a photodetector fabricated on a $200-\mathrm{nm} \mathrm{SiO}_{2}$ substrate as calculated by the finite-difference-time-domain simulation. An overview of the spectral selectivity upon changing the oxide thickness is shown in Figure 4c.

In contrast to the previously demonstrated graphene-semiconductor phototransistor, our phototransistor relies on the photon absorption 
a
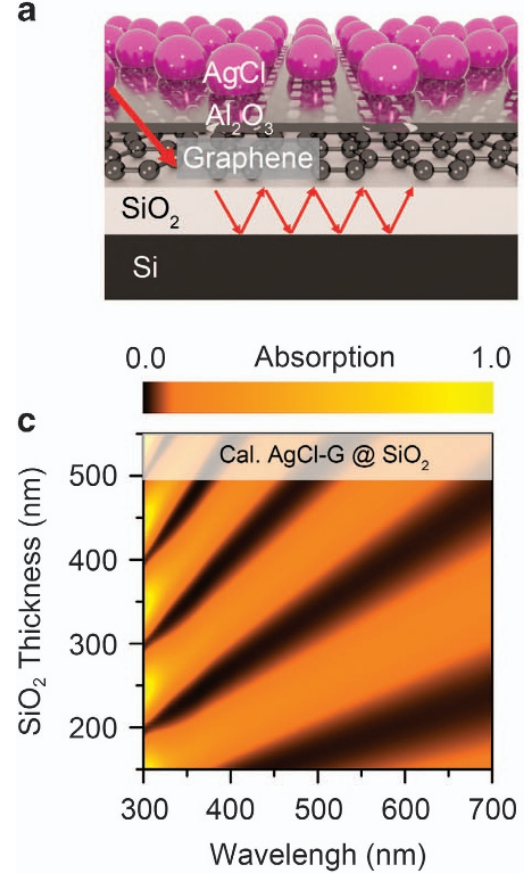

b

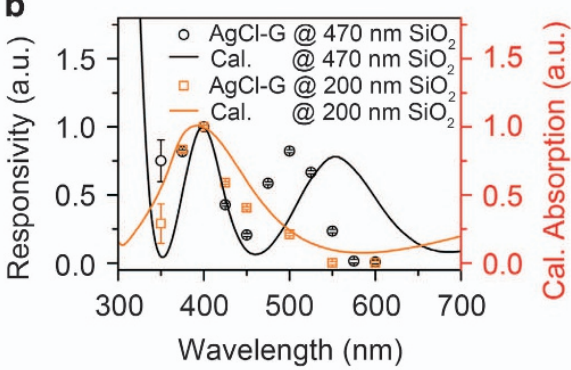

d

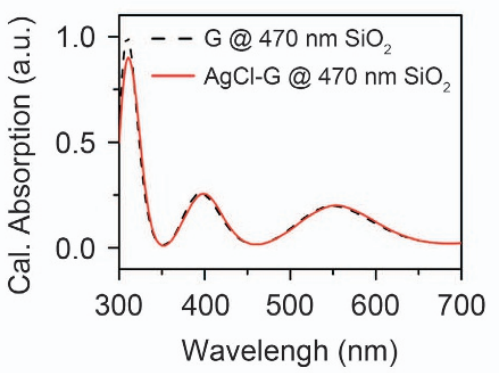

Figure 4 Spectral selectivity modulation. (a) Illustration of multiple reflections within the dielectric layer beneath the monolayer graphene. (b) The experimental wavelength-dependent responsivity of $\mathrm{AgCl}-\mathrm{G}$ on thermal oxide substrates at $V_{\mathrm{SD}}=1 \mathrm{~V}$ and $V_{\mathrm{G}}=0 \mathrm{~V}$ (black circles for 470 -nm $\mathrm{SiO}_{2}$ and brown squares for 200-nm $\mathrm{SiO}_{2}$, left axis) and corresponding $\mathrm{FDTD}^{-c a l c u l a t e d}$ absorption spectra (black line for $470-\mathrm{nm} \mathrm{SiO}_{2}$ and brown line for 200-nm $\mathrm{SiO}$, right axis). All of the data were normalized to the responsivity at $400 \mathrm{~nm}$. (c) Absorption intensity map for AgCl-G as a function of both the excitation wavelength and the $\mathrm{SiO}_{2}$ thickness. (d) The calculated absorption of $\mathrm{AgCl}-\mathrm{G}$ and bare graphene on $470-\mathrm{nm} \mathrm{SiO} 2$ after normalization to the peak value of bare graphene. FDTD, finite-difference-time-domain.

in the graphene layer rather than in the semiconductor. As shown in Figure $4 \mathrm{~d}$, the simulated absorption spectra of $\mathrm{AgCl}-\mathrm{G}$ and bare graphene match well with each other in the visible region, indicating that the light absorption in $\mathrm{AgCl}$ is negligible for the $\mathrm{AgCl}-\mathrm{G}$ heterostructure. The power absorption ratio between graphene $\left(P_{\text {graphene }}\right)$ and $\mathrm{AgCl}\left(P_{\mathrm{AgCl}}\right)$ is 126:1 for 400 -nm incident wavelength and even higher for $500 \mathrm{~nm}$ (246:1) (Supplementary Figure 12), which again confirms the distinct sensing mechanism of the AgCl-G hybrid phototransistor.

\section{DISCUSSION}

Photoconductive gain is a key figure of merit for a photodetector. Based on the mobility value in AgCl-G, the transit time $\left(\tau_{\text {transit }}=L^{2} \mu^{-1} V_{\mathrm{SD}}^{-1}\right)$ of the carriers is $\sim 2.76 \mathrm{~ns}^{10}$ Therefore, the photoconductive gain is estimated to be $\sim 3.04 \times 10^{9}$ by adopting $\tau_{\text {lifetime }}=8.4 \mathrm{~s}$ (Figure $1 \mathrm{~b}$, $\sim 50 \%$ photocurrent decay), which is over 30 times larger than the value of a PbS QD-graphene hybrid photodetector. ${ }^{9}$ Because the photoconductive gain scales linearly with the mobility of graphene, we can expect that the responsivity can be further improved by adopting boron nitride ${ }^{27}$ or a self-assembled monolayer-coated oxide ${ }^{10}$ as the substrate. One drawback of our device is that the response speed is slower compared to the previously demonstrated graphene hybrid photodetector primarily due to its slow turnoff speed. This is understandable because the $\mathrm{AgCl}$ nanoparticles in our device are in situ synthesized by gas phase halogenation in which more deep trap states are present on the $\mathrm{AgCl}$ surface compared to solution-synthesized QDs, which significantly slows down the charge released in the dark. To facilitate the charge releasing, we applied a positive reset pulse at the gate electrode, which lowered the Fermi level of graphene and promoted the charge extraction from the trap states in $\mathrm{AgCl}$ to graphene. ${ }^{9}$ As shown in Supplementary Figure 13, the transit time of our photodetector can be effectively reduced to $2.3 \mathrm{~s}$ by introducing the reset pulse. We expect that by improving the synthesis process and introducing surface passivation to the $\mathrm{AgCl}$, a further improved response speed can be achieved. Moreover, $\mathrm{ZnO}$ has a band structure that is similar to $\mathrm{AgCl}$ (bandgap $E_{\mathrm{g}}^{\mathrm{ZnO}}=3.2 \mathrm{eV}, E_{\mathrm{g}}^{\mathrm{AgCl}}=3.25 \mathrm{eV}$; conduction band minimum $E_{\mathrm{c}}^{\mathrm{ZnO}}=-4.19 \mathrm{eV}, E_{\mathrm{c}}^{\mathrm{AgCl}}=-4.3 \mathrm{eV}$ and valence band maximum $\left.E_{\mathrm{V}}^{\mathrm{ZnO}}=-7.39 \mathrm{eV}, E_{\mathrm{V}}^{\mathrm{AgCl}}=-7.55 \mathrm{eV}\right) ;^{28-31}$ however, only an ultraviolet response was observed in a $\mathrm{ZnO}$-graphene hybrid. ${ }^{8,10,11}$ This fact indicated the vital role of the $\mathrm{AgCl} / \mathrm{Ag}^{0}$ redox couple in our photodetector. To evaluate the noise characteristics of our photodetector, the noise-equivalent-power, which is the optical power that yields a unity signal-to-noise ratio for a given bandwidth, was estimated by analyzing the noise in the dark current (Supplementary Figure 14). ${ }^{32}$ A noise-equivalent-power of $\sim 10^{-14} \mathrm{~W} \mathrm{~Hz}^{-1 / 2}$ can be achieved at a frequency of $1 \mathrm{~Hz}$ by dividing the noise spectral density by the responsivity ( $7.3 \times 10^{4} \mathrm{AW}^{-1}$ at $500 \mathrm{~nm}$, Figure $\left.3 \mathrm{~b}\right)$.

In summary, we demonstrated a hybrid graphene phototransistor for sub-bandgap photon sensing by utilizing the electrochemical redox half-reaction of silver chloride as a reversible carrier reservoir. Specifically, an ultrahigh photoconductive gain and photoresponsivity far beyond the absorption band edge of $\mathrm{AgCl}$ was achieved. Our experiment and simulation results reveal that graphene can serve as a light absorbing material in a hybrid graphene phototransistor in which the spectral response is no longer limited by the integrated semiconducting material. This study not only presents a new paradigm of sub-bandgap absorption in semiconductor-graphene heterostructures but also inspires the broader application of the electrochemical redox half-reaction in other optoelectronic devices. 


\section{CONFLICT OF INTEREST}

ZX, JC and JT have filed a US patent based on this work (application no. $62 / 346,706)$. The remaining authors declare no confict of interest.

\section{ACKNOWLEDGEMENTS}

This work was supported in part by the Hong Kong Research Grants Council (RGC) General Research Fund (GRF17303015, GRF17305917, ECS27300814), the University Grant Council (Contract No. AoE/ P-04/08), the URC Strategic Research Theme on Clean Energy (University of Hong Kong), and the URC Strategic Research Theme on New Materials (University of Hong Kong).

Author contributions: ZX, JC and JT conceived the project. ZX, JC, JW and YC performed the sample preparation and device fabrication. ZX, JC, ZS and SX performed the photocurrent measurements. ZX, XL and AK performed the KPFM measurements. ZX and WL performed the FDTD simulation. JB performed the XPS measurements. GL and MH performed the absorbance measurements. ZX and JT co-wrote the paper with all authors contributing to the discussion and preparation of the manuscript.

\section{PUBLISHER'S NOTE}

Springer Nature remains neutral with regard to jurisdictional claims in published maps and institutional affiliations.

1 Lemme, M. C., Koppens, F. H. L., Falk, A. L., Rudner, M. S., Park, H., Levitov, L. S. \& Marcus, C. M. Gate-activated photoresponse in a graphene $p-n$ junction. Nano Lett. 11, 4134-4137 (2011).

2 Mueller, T., Xia, F. \& Avouris, P. Graphene photodetectors for high-speed optical communications. Nat. Photon. 4, 297-301 (2010).

3 Tielrooij, K. J., Piatkowski, L., Massicotte, M., Woessner, A., Ma, Q., Lee, Y., Myhro, K. S., Lau, C. N., Jarillo Herrero, P., van Hulst, N. F. \& Koppens, F. H.L. Generation of photovoltage in graphene on a femtosecond timescale through efficient carrier heating. Nat. Nanotechnol. 10, 437-443 (2015).

4 Liu, Y., Wang, F., Wang, X., Wang, X., Flahaut, E., Liu, X., Li, Y., Wang, X., Xu, Y. Shi, Y. \& Zhang, R. Planar carbon nanotube-graphene hybrid films for high-performance broadband photodetectors. Nat. Commun. 6, 9589 (2015).

5 Lee, Y., Kwon, J., Hwang, E., Ra, C.-H., Yoo, W. J., Ahn, J.-H., Park, J. H. \& Cho, J. H. High-performance perovskite-graphene hybrid photodetector. Adv. Mater. 27, 41-46 (2015)

6 Wang, Y., Zhang, Y., Lu, Y., Xu, W., Mu, H., Chen, C., Qiao, H., Song, J., Li, S., Sun, B., Cheng, Y.-B. \& Bao, Q. Hybrid graphene-perovskite phototransistors with ultrahigh responsivity and gain. Adv. Opt. Mater. 3, 1389-1396 (2015).

7 Chen, S.-Y., Lu, Y.-Y., Shih, F.-Y., Ho, P.-H., Chen, Y.-F., Chen, C.-W., Chen, Y.-T. \& Wang, W.-H. Biologically inspired graphene-chlorophyll phototransistors with high gain. Carbon 63, 23-29 (2013).

8 Chang, H., Sun, Z., Ho, K. Y.-F., Tao, X., Yan, F., Kwok, W.-M. \& Zheng, Z. A highly sensitive ultraviolet sensor based on a facile in situ solution-grown $\mathrm{ZnO}$ nanorod/ graphene heterostructure. Nanoscale 3, 258-264 (2011).

9 Konstantatos, G., Badioli, M., Gaudreau, L., Osmond, J., Bernechea, M., de Arquer, F. P.G., Gatti, F. \& Koppens, F. H.L. Hybrid graphene-quantum dot phototransistors with ultrahigh gain. Nat. Nanotechnol. 7, 363-368 (2012).

10 Shao, D., Gao, J., Chow, P., Sun, H., Xin, G., Sharma, P., Lian, J., Koratkar, N. A \& Sawyer, S. Organic-inorganic heterointerfaces for ultrasensitive detection of ultraviolet light. Nano Lett. 15, 3787-3792 (2015).

11 Guo, W., Xu, S., Wu, Z., Wang, N., Loy, M. M.T. \& Du, S. Oxygen-Assisted Charge Transfer Between ZnO quantum dots and graphene. Small 9, 3031-3036 (2013).

12 Shin, D. H., Kim, S., Kim, J. M., Jang, C. W., Kim, J. H., Lee, K. W., Kim, J., Oh, S. D., Lee, D. H., Kang, S. S., Kim, C. O., Choi, S.-H. \& Kim, K. J. Graphene/Si-quantum-dot heterojunction diodes showing high photosensitivity compatible with quantum confinement effect. Adv. Mater. 27, 2614-2620 (2015).
13 Zhang, D. Y., Gan, L., Cao, Y., Wang, Q., Qi, L. M. \& Guo, X. F. Understanding charge transfer at PbS-decorated graphene surfaces toward a tunable photosensor. Adv. Mater. 24, 2715-2720 (2012).

14 Massicotte, M., Schmidt, P., Vialla, F., Watanabe, K., Taniguchi, T., Tielrooij, K. J. \& Koppens, F. H.L. Photo-thermionic effect in vertical graphene heterostructures. Nat. Commun. 7, 12174 (2016).

15 Wang, X. M., Cheng, Z. Z., Xu, K., Tsang, H. K. \& Xu, J. B. High-responsivity graphene/ silicon-heterostructure waveguide photodetectors. Nat. Photon. 7, 888-891 (2013).

16 Rodriguez-Nieva, J. F., Dresselhaus, M. S. \& Song, J. C.W. Enhanced thermionicdominated photoresponse in graphene Schottky junctions. Nano Lett. 16, 6036-6041 (2016).

17 Zheng, B. Y., Zhao, H., Manjavacas, A., McClain, M., Nordlander, P. \& Halas, N. J. Distinguishing between plasmon-induced and photoexcited carriers in a device geometry. Nat. Commun. 6, 7797 (2015).

18 Vanýsek, P. CRC Handbook of Chemistry and Physics, 96th edn (CRC Press, 2015).

19 Song, S. M., Park, J. K., Sul, O. J. \& Cho, B. J. Determination of work function of graphene under a metal electrode and its role in contact resistance. Nano Lett. 12 3887-3892 (2012).

20 Yu, Y.-J., Zhao, Y., Ryu, S., Brus, L. E., Kim, K. S. \& Kim, P. Tuning the graphene work function by electric field effect. Nano Lett. 9, 3430-3434 (2009).

21 Panchal, V., Pearce, R., Yakimova, R., Tzalenchuk, A. \& Kazakova, O. Standardization of surface potential measurements of graphene domains. Sci. Rep. 3, 2597 (2013).

22 Knight, M. W., Sobhani, H., Nordlander, P. \& Halas, N. J. Photodetection with active optical antennas. Science 332, 702-704 (2011).

23 Schwierz, F. Graphene transistors. Nat. Nanotechnol. 5, 487-496 (2010).

24 Engel, M., Steiner, M., Lombardo, A., Ferrari, A. C., Löhneysen, H.v., Avouris, P. \& Krupke, R. Light-matter interaction in a microcavity-controlled graphene transistor. Nat. Commun. 3, 906 (2012).

25 Gan, X. T., Shiue, R. J., Gao, Y. D., Meric, I., Heinz, T. F., Shepard, K., Hone, J. Assefa, S. \& Englund, D. Chip-integrated ultrafast graphene photodetector with high responsivity. Nat. Photon. 7, 883-887 (2013).

26 Echtermeyer, T. J., Britnell, L., Jasnos, P. K., Lombardo, A., Gorbachev, R. V., Grigorenko, A. N., Geim, A. K., Ferrari, A. C. \& Novoselov, K. S. Strong plasmonic enhancement of photovoltage in graphene. Nat. Commun. 2, 458 (2011).

27 Gannett, W., Regan, W., Watanabe, K., Taniguchi, T., Crommie, M. F. \& Zettl, A. Boron nitride substrates for high mobility chemical vapor deposited graphene. Appl. Phys. Lett. 98, 242105 (2011).

28 Bose, D. N. \& Govindacharyulu, P. A. Physics of silver halides and their applications. Bull. Mater. Sci. 2, 221-231 (1980).

29 El Halouani, F. \& Deschanvres, A. Interfaces semi-conducteur-electrolyte: correlations entre le potentiel de bande plate et les echelles d'electronegativite. Mater. Res. Bull. 17, 1045-1052 (1982).

30 Sumi, S., Watanabe, T., Fujishima, A. \& Honda, K. Effect of $\mathrm{Cl}$ - and $\mathrm{Br}$-ions and $\mathrm{pH}$ on the flatband potentials of silver halide sheet crystal electrodes. Bull. Chem. Soc. Jpn. 53, 2742-2747 (1980).

31 Bauer, R. S. \& Spicer, W. E. Silver-halide valence and conduction states: temperaturedependent ultraviolet-photoemission studies. Phy. Rev. B 14, 4539-4550 (1976).

32 Liu, C. H., Chang, Y. C., Norris, T. B. \& Zhong, Z. H. Graphene photodetectors with ultra-broadband and high responsivity at room temperature. Nat. Nanotechnol. 9, 273-278 (2014).

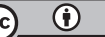

This work is licensed under a Creative Commons Attribution 4.0 International License. The images or other third party material in this article are included in the article's Creative Commons license, unless indicated otherwise in the credit line; if the material is not included under the Creative Commons license, users will need to obtain permission from the license holder to reproduce the material. To view a copy of this license, visit http:// creativecommons.org/licenses/by/4.0/

(C) The Author(s) 2017

Supplementary Information accompanies the paper on the NPG Asia Materials website (http://www.nature.com/am) 